\title{
Impact of testicular shielding in liposarcoma to scrotum by using radio-photoluminescence glass dosimeter (RPLGD): a case report
}

\author{
Puntiwa Oonsiri, MSc ${ }^{1}$, Kitwadee Saksornchai, MD', Sivalee Suriyapee, MEng ${ }^{2}$ \\ 'Department of Radiation Oncology, King Chulalongkorn Memorial Hospital, The Thai Red Cross Society, Bangkok; \\ ${ }^{2}$ Faculty of Medicine, Chulalongkorn University, Bangkok, Thailand
}

Radiation protection in the scrotum to reduce the risk of genetic effect in the future is very important. This study aimed to measure the scrotal dose outside the treatment fields by using the radio-photoluminescence glass dosimeter (RPLGD). The characteristics of RPLGD model GD-302M were studied. Scattered dose to scrotum was measured in one liposarcoma case with the prescribed dose of 60 Gy. RPLGDs were placed in three different locations: one RPLGD was positioned at the posterior area which closer to the scrotum, and the other two RPLGDs were placed between the penis and the scrotum. Three RPLGDs were employed in each location. The scattered doses were measured in every fraction during the whole course of treatment. The entire number of 100 RPLGDs showed the uniformity within $\pm 2 \%$. The signal from RPLGD demonstrated linear proportion to the radiation dose $(r=0.999)$. The relative energy response correction factor was 1.05. The average scrotal dose was $4.1 \pm 0.9$ cGy per fraction. The results presented a wide range since there was a high uncertainty during RPLGD placement. The total scrotal dose for the whole course of treatment was $101.9 \mathrm{cGy}$ ( $1.7 \%$ of the prescribed dose). The RPLGD model GD-302M could be used to measure scattered dose after applying the relative energy correction factor.

Keywords: Radio-photoluminescent glass dosimeter (RPLGD), Scrotal dose, Testicular shielding

\section{Introduction}

The unintentional dose out of the treatment fields in radiotherapy comes from the internal scattered within the patient body, the collimator scatters, and the leakage [1]. For male patients in the reproductive age who undergo radiotherapy treatment, such unintentional dose to scrotum becomes a great concern since this organ is highly responsive to radiation. The side effect of low dose scattered radiation could be potentially affecting the fertility of patient as well as inducing unwanted genetic effects in the future. Furthermore, a cumulative scrotal dose more than 200 cGy may lead into permanent sterility. The necessity for employing an adequate shielding to male patients has been recommended by Ravichandran et al. [2] and Singhal et al. [3] to protect scrotal dose to receive a dose less than $2 \%$ of the prescribed dose.

Several studies to measure the scrotal dose using thermoluminescence dosimeter (TLD) have been reported [3,4]. Some testicular shielding made from low melting alloy or Cerrobend are commonly used with an excellent outcome to

Received 24 March 2018, Revised 07 May 2018, Accepted 07 June 2018.

Correspondence: Puntiwa Oonsiri, MSc, Department of Radiation Oncology, King Chulalongkorn Memorial Hospital, The Thai Red Cross Society, Bangkok, Thailand. Tel: +662-256-4334, Fax: +662-251-4661, E-mail: nim_1000d@hotmail.com

(c) This is an Open Access article distributed under the terms of the Creative Commons Attribution Non-Commercial License (http://creativecommons.org/ licenses/by-nc/4.0/) which permits unrestricted non-commercial use, distribution, and reproduction in any medium, provided the original work is properly cited.

www.e-roj.org 
reduce scrotal dose below than $2 \%$ of the prescribed dose. In our center, the CIVCO testicular shielding (CIVCO Radiotherapy, Orange City, IA, USA) is used routinely. This shielding is fabricated from the lead. Nevertheless, no literature so far has been published about the use of this shielding for protecting scrotum.

Currently, the radio-photoluminescence glass dosimeter (RPLGD) is a new trend of passive dosimeter which is increasingly used rather than TLD. Several benefits of RPLGD as confirmed by Hsu et al. [5] such as repeatable readout, good reproducibility, wide measurable dose range, less energy dependence, and less fading effect have brought the use of RPLGD become more superior than TLD. Another dosimeter which is widely used for in vivo dosimetry is optically stimulated luminescence (OSL). However, in comparison to RPLGD, OSL presents a drawback in regards to the dose response which strongly depends on its crystal growth [6] Other pitfalls are related to the optical fiber used in front of the PMT [7], the stimulation intensities [8], and the dose history of the OSL [9]. A study from Knezevic et al. [10] concluded that the RPLGD was more suitable than TLD and OSL since they found the RPLGD did not exhibit supra-linearity for a higher dose. Moreover, RPLGD did not require individual sensitivity correction factors like TLD and OSL.

This study was based on the case report where we reported a male patient who underwent radiotherapy treatment for liposarcoma whom he wished to still have descendant after completing the treatment. We aimed to determine his scrotal dose during the whole course of treatment using RPLGD.

\section{Case Report}

\section{Glass dosimeter system}

The RPLGD system consists of a glass detector model 302M (GD-302M), the FGD-1000 dosimeter reader, and an electric furnace (AGC Techno Glass Co. Ltd, Shizuoka, Japan). The glass element series FD-7 is composed of several components by weight: $\mathrm{Na}$ (11\%), P (31.55\%), $\mathrm{O}$ (51.16\%), Al (6.12\%), and Ag $(0.17 \%)$. The effective atomic number is 12.04 . The cylindrical dimensions of GD-302M are $1.5 \mathrm{~mm}$ diameter and $12 \mathrm{~mm}$ length (2.8 $\mathrm{mm} \times 13 \mathrm{~mm}$ area including the holder size). The process for each experiment is elaborated as follows: (1) annealing $\left(400^{\circ} \mathrm{C}\right.$ for 1 hour) to initialize the integrated dose value in glass element, (2) readout of initial dose value for splinter, stain or cloud checking, (3) beam irradiation, (4) preheating $\left(70^{\circ} \mathrm{C}\right.$ for 30 minutes) to bring the electron to the correct color center and to stabilize the luminescent signal of the RPLGD before reading, and (5) read out of accumulated value at the room temperature. The dosimetric characteristics of glass dosimeter such as uniformity, the linearity, and the relative energy response were observed before measuring the scrotal dose.

\section{Dosimetric characteristics of glass dosimeter}

Firstly, we performed calibration of $6 \mathrm{MV}$ photon beams from Varian Clinac 21EX (Varian Oncology System, Palo Alto, CA, USA). A prescribed dose of 100 cGy was delivered to a solid water phantom (Gammex-RMI Inc., Middleton, WI, USA) and the ionization chamber FC-65G (IBA Dosimetry GmbH, Schwarzenbruck, Germany) along with the DOSE-I electrometer (IBA Dosimetry GmbH, Schwarzenbruck, Germany) were utilized to measure the collected charge. The field size was set up to $20 \mathrm{~cm} \times 20 \mathrm{~cm}$ and source to surface distance (SSD) was adjusted to $100 \mathrm{~cm}$. The depth of measurement was set to 5 $\mathrm{cm}$.

Secondly, the calibration of glass detector was performed under the same condition. The RPLGDs were positioned similarly to the calibration condition for characteristics measurement as shown in Fig. 1A. Except at $5 \mathrm{~cm}$ depth, a bolus with $1 \mathrm{~cm}$ thickness was placed at the inferior and superior part of RPLGD to reduce the air gap effect when using solid water phantom. For the uniformity study, the RPLGDs were arranged in 5 rows and 20 columns in the middle of the field for single irradiation of 100 cGy. The automatic reader FGD-1000 was employed to read the signal. The signals were read five times for each dosimeter. For the linearity, the doses at 1, 3, 5, 10, 50, and 100 cGy were delivered to five RPLGDs.

Lastly, the $0.38 \mathrm{MeV} \gamma$-rays from Ir-192 source was inserted into the center of the cylindrical PMMA phantom for brachytherapy to represent the energy of scattered radiation which is typically ranging from $0.2 \mathrm{MeV}$ to $0.6 \mathrm{MeV}$ according to AAPM TG-158 reported [11]. Two RPLGDs were inserted in the cavity plug at the peripheral side of the cylindrical PMMA phantom. The ionization chamber FC-65G was placed in the opposing side of the glass detectors and connected to the DOSE-I electrometer (IBA Dosimetry $\mathrm{GmbH}$ ) to measure radiation dose at the same distance and time to validate the accuracy of measured dose from RPLGDs. The setup position among RPLGD, ionization chamber FC-65G, and the Ir-192 source is depicted in Fig. 1B.

\section{Scrotal dose measurement}

The Institutional Review Board of Faculty of Medicine, 
A

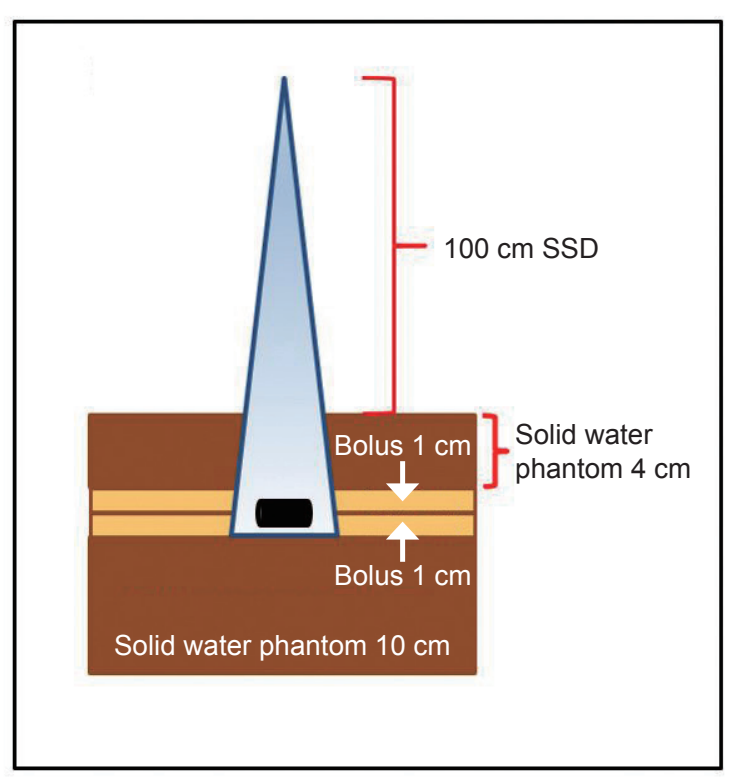

B

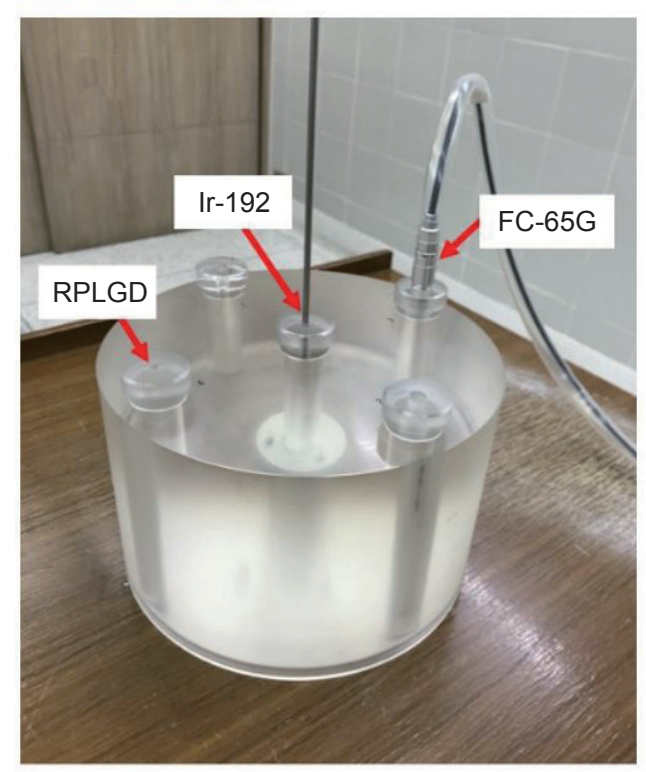

Fig. 1. (A) The setup position of radio-photoluminescence glass dosimeter (RPLGD) under calibration condition for $6 \mathrm{MV}$ photon beams and (B) the setup position of RPLGD, Ir-192, and FC-65G inside the cylindrical PMMA phantom for brachytherapy.

Chulalongkorn University has approved our study to measure the scrotal dose of 41 -year-old male patient (IRB No. 085/61). His case was liposarcoma mass on the right thigh. He underwent wide excision and the results were grade I, $15 \times$ $9.5 \times 8.0 \mathrm{~cm}^{3}$ volume, no lymphovascular invasion, uninvolved resection margin, and well-differentiated liposarcoma. He was referred to our clinic for postoperative radiation therapy. The simulation process was performed in a supine position where we instructed him to spread his left thigh as much as possible to keep the distance of scrotum away from the irradiated field. The computed tomography (CT) images were acquired using Siemens Somatom Definition AS Open 64 slices (Siemens, Erlangen, Germany) in 3-mm slice thickness. The radiation oncologist contoured the scrotum before dose calculation was performed in Eclipse treatment planning version 11.0.31 (Varian Oncology Systems). The AP/PA treatment at the right thigh at $15 \mathrm{~mm} \times 30 \mathrm{~cm}$ field size was planned with $6 \mathrm{MV}$ photon beams from Varian TrueBEAM System (Varian Oncology Systems). The total dose of 60 Gy was prescribed in 30 fractions for 6 weeks. The testicular shielding and stand (CIVCO Radiotherapy) were incorporated into a patient for protecting the scrotal area during treatment. The scattered dose to the scrotum was measured by using the RPLGD. The position of RPLGD was approximately $7 \mathrm{~cm}$ from the proximal edge of the treatment field. The RPLGDs were placed in three positions inside the testicular shielding. One RPLGD was positioned at the posterior area which closer to the scrotum, and the other two RPLGDs were placed between the penis and the scrotum as illustrated in Fig. 2.

\section{Discussion}

\section{Dosimetric characteristics of glass dosimeter}

The uniformity of 100 RPLGDs was represented in the relative response, the readout of each RPLGD normalized to the average of hundred RPLGDs readout, where its average was $1.00 \pm 0.02$. The batch uniformity was the coefficient of variation which defined by the standard deviation divided by the average reading. The batch uniformity in our study was $\pm 2.0 \%$ which was agreeable to Hsu et al. [12] study where their result was $\pm 1.5 \%$. To evaluate the linearity response, the readout of each RPLGD was plotted according to the dose setting as depicted in Fig. 3. The RPLGD signal demonstrated a linear proportion to the radiation dose ranging from $1 \mathrm{cGy}$ to $100 \mathrm{cGy}(r=0.999)$. Our finding was consistent with the results from Hsu et al. [12] and Kihong Son et al. studies [12,13]. For the relative energy response, the readout value of the RPLGD at $0.38 \mathrm{MeV}$ was normalized to the average signal of 6 MV photon beams under the same radiation dose. An increase of luminescence signal with increasing signal due to photoelectric effect after irradiation to GD-302M using low energy photons was discovered in our study [14]. Afterward, the 

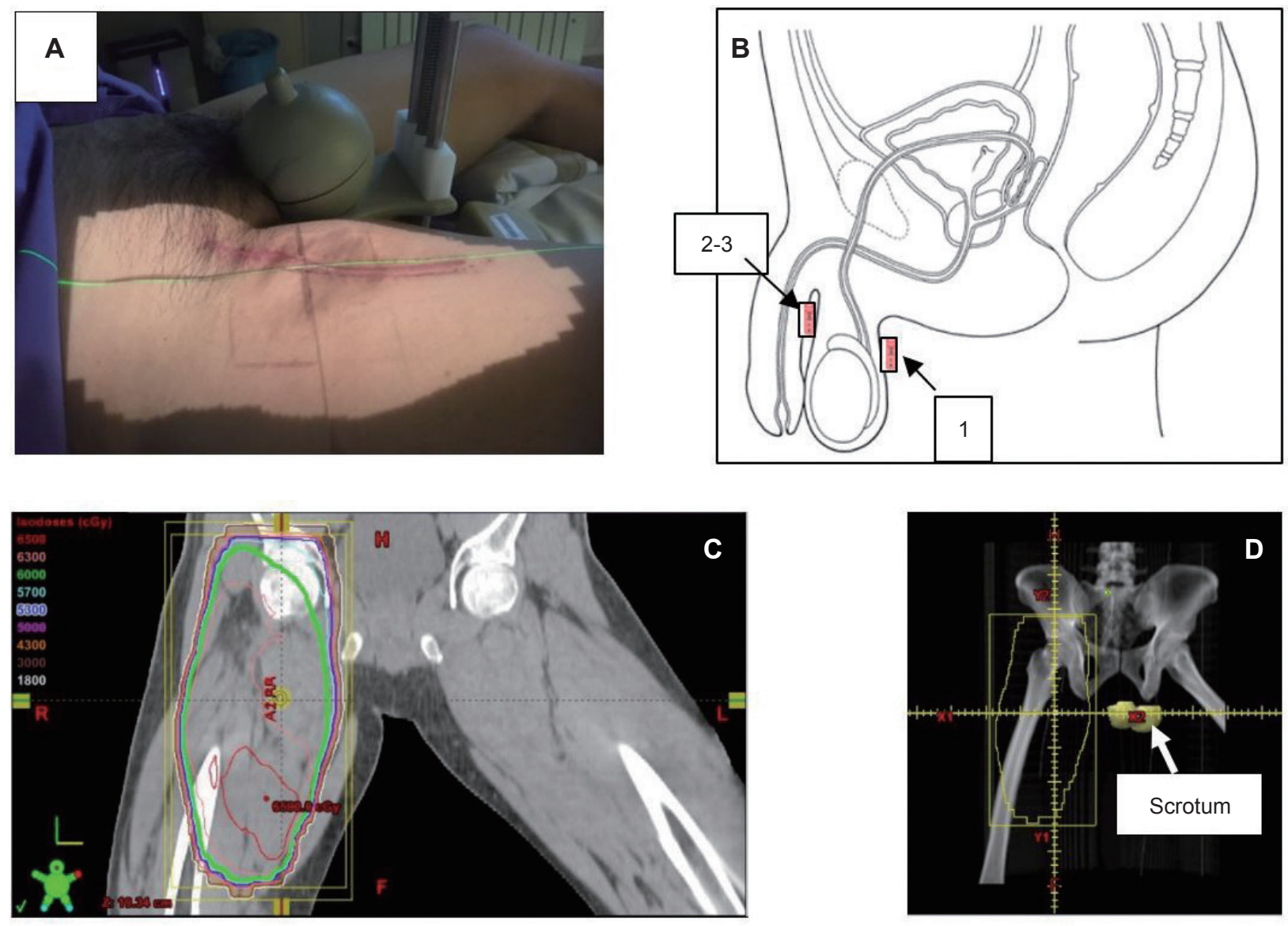

Fig. 2. (A) The setup of testicular shield for liposarcoma case. (B) The radio-photoluminescence glass dosimeter (RPLGD) placement inside the testicular shield. (C, D) The irradiation field for liposarcoma case: coronal plane and digital reconstructed radiograph.

relative energy response correction factor of 1.05 was applied for scrotal dose measurement in every fraction.

\section{Scrotal dose measurement}

Since the use of testicular shield produced a streak artifact in the image, the electronic portal imaging device (EPID) was selected as an image verification tool rather than using OnBoard Imager (OBI) system. The EPID was used in every fraction while verification using CBCT was totally made in 9 sessions taken for every fraction in the first week and weekly afterward. The imaging doses during treatment verification were taken into account as the additional doses to the scrotum. Pyone et al. [15] reported the EPID and CBCT dose estimation to the scrotum in our TrueBEAM system by using TLD. The scrotal dose from image verification procedure was approximately $5 \mathrm{mGy}$ and $3 \mathrm{mGy}$ in a single session for EPID and CBCT, respectively.

The average scrotal dose for each fraction is given in Fig.

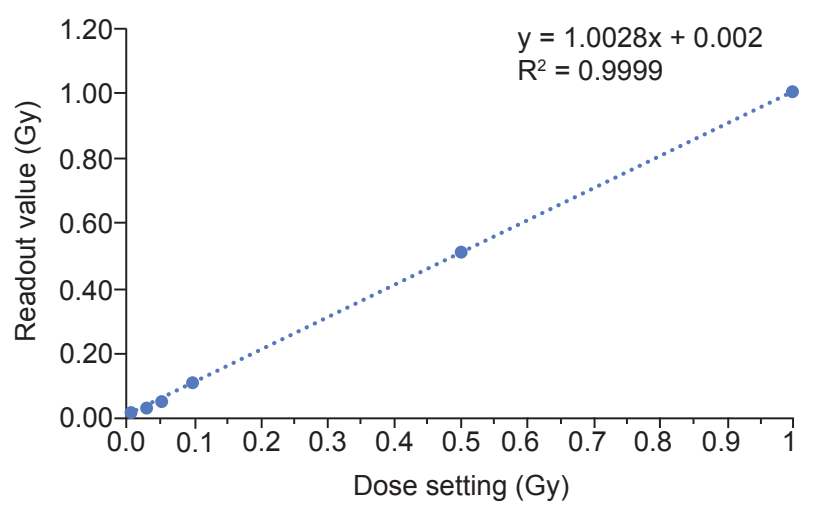

Fig. 3. The linearity response of radio-photoluminescence glass dosimeters.

4. In this work, the scrotal dose measurement from RPLGD was not separated from the imaging dose during verification procedure as well as the scattered radiation dose out-of-field. 


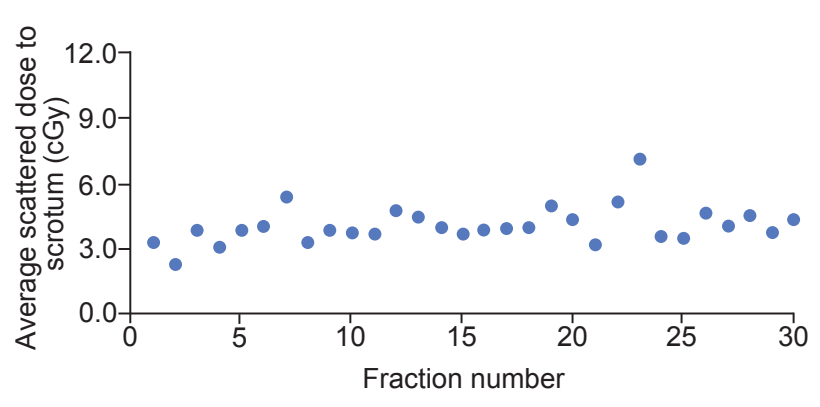

Fig. 4. The average scattered dose to the scrotum for each fraction.

Therefore, the relative energy response correction factor was assumed to be similar for the energy of scattered radiation and energy of imaging verification. Once the single value for relative energy response correction factor was applied, the highest dose of $7.1 \mathrm{cGy}$ was detected in the 23th fraction. At that moment, the patient suffered edema and leg pain. Hence, the radiation technologist was unable to move the scrotum away from the treatment fields like previous fractions. Overall, the average scrotal dose became $4.1 \pm 0.9 \mathrm{cGy}$ per fraction (range, 2.3 to $7.1 \mathrm{cGy}$ ). High uncertainty in the placement of glass dosimeters was identified as the main reason for significant fluctuation at every position that received the highest dose. The total scrotal dose for the whole course of treatment was $101.9 \mathrm{cGy}$ from $60 \mathrm{~Gy}$ of the total dose (1.7\% of the prescribed dose) including imaging verification dose of $17.7 \mathrm{cGy}$ ( $0.3 \%$ of the prescribed dose). The result was higher than a study from Ravichandran et al. [2], where they found the scrotal dose was $0.8 \%$ of the prescribed dose. Higher dose in our work was corresponded to the shorter distance of the irradiation field to scrotal area compared to the study of Ravichandran et al. [2]. In our study, the distance was 7 $\mathrm{cm}$, while Ravichandran et al. [2] set the distance of $14 \mathrm{~cm}$. However, the total scrotal dose was still in the limit of $2 \%$ of the prescribed dose which has been recommended by Purdy et al. [4]. Our work showed the testicular shielding made by lead was suitable to protect the scrotum from low energy doses associated with the collimator leakage and the internal scattered dose.

The mean scrotal dose at $7 \mathrm{~cm}$ from the edge of the treatment field was $142.7 \mathrm{cGy}$ which was calculated by Eclipse treatment planning system. However, the calculated dose was not compared to the measured dose since the CT images for dose calculation were acquired without the testicular shield. As previously mentioned, the $\mathrm{CT}$ images presented more streak artifacts. Therefore, the calculated dose in scrotum was unable to be determined properly.

According to our results, there were two factors attributed to the small amount of scattered dose to the scrotum. The first was the use of testicular shielding and the second was patient's position to keep the distance of the scrotum far away from the treatment field by spreading his left thigh during treatment.

The RPLGD demonstrates good capability in regards to its uniformity and linearity. Moreover, each detector has a small size and easy to identify from its ID as can be seen at the edge of RPLGD. These advantages make the use of RPLGD is considered state-of-the-art yet feasible for in vivo dose measurement. However, attention to the relative energy response correction factor should be paid when the scattered dose out-of-field is taken into account. Our study utilized the RPLGD for scrotal dose measurement for out of irradiation field. The total scrotal dose was $1.7 \%$ of the prescribed dose with contribution from imaging dose verification of $0.3 \%$. Our finding confirms that the RPLGD model GD-302M could be employed to measure scrotal dose after the relative energy correction factor is applied.

\section{Conflict of Interest}

No potential conflict of interest relevant to this article was reported.

\section{Acknowledgments}

The authors would like to express the most sincere gratitude to Miss Woraya Nguanthean, radiation therapy technologist of King Chulalongkorn Memorial Hospital for glass detectors placement.

\section{References}

1. Clifford Chao KS, Perez CA, Brady LW. Radiation oncology: management decisions. 2nd ed. Philadelphia, PA: Lippincott Williams \& Wilkins; 2002.

2. Ravichandran R, Binukumar JP, Kannadhasan S, Shariff MH, Ghamrawy KE. Testicular shield for para-aortic radiotherapy and estimation of gonad doses. J Med Phys 2008;33:158-61.

3. Singhal MK, Kapoor A, Singh D, et al. Scattered radiation to gonads: role of testicular shielding for para-aortic and homolateral illiac nodal radiotherapy. J Egypt Natl Canc Inst 2014;26(2):99-101. 
4. Purdy JA, Stiteler RD, Glasgow GP, Mill WB. Gonadal shield. Radiology 1975;117:226.

5. Hsu SM, Yeh SH, Lin MS, Chen WL. Comparison on characteristics of radiophotoluminescent glass dosemeters and thermoluminescent dosemeters. Radiat Prot Dosimetry 2006;119:327-31.

6. Yukihara EG, Whitley VH, McKeever SW, Akselrod AE, Akselrod MS. Effect of high-dose irradiation on the optically stimulated luminescence of Al203:C. Radiat Meas 2004;38:317-30.

7. Yukihara EG, McKeever SW. Spectroscopy and optically stimulated luminescence of Al203:C using time-resolved measurements. J Appl Phys 2006;100:083512.

8. Yukihara EG, McKeever SW. Optically stimulated luminescence (OSL) dosimetry in medicine. Phys Med Biol 2008;53:R351-79.

9. Edmund JM, Andersen CE, Marckmann CJ, Aznar MC, Akselrod MS, Botter-Jensen L. CW-OSL measurement protocols using optical fibre Al203:C dosemeters. Radiat Prot Dosimetry 2006;119:368-74.

10. Knezevic Z, Stolarczyk L, Bessieres I, Bordy JM, Miljanic S, Olko P. Photon dosimetry methods outside the target volume in radiation therapy: Optically stimulated luminescence (OSL), thermoluminescence $(\mathrm{TL})$ and radiophotoluminescence $(\mathrm{RPL})$ dosimetry. Radiat Meas 2013;57:9-18.

11. Kry SF, Bednarz B, Howell RM, et al. AAPM TG 158: Measurement and calculation of doses outside the treated volume from external-beam radiation therapy. Med Phys 2017:44:e391-e429.

12. Hsu SM, Yang HW, Yeh TC, et al. Synthesis and physical characteristics of radiophotoluminescent glass dosimeters. Radiat Meas 2007;42:621-4.

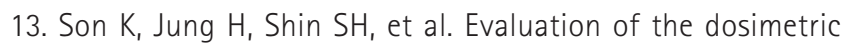
characteristics of a radiophotoluminescent glass dosimeter for high-energy photon and electron beams in the field of radiotherapy. Radiat Meas 2011;46:1117-22.

14. Huang DY, Hsu SM. Radio-photoluminescence glass dosimeter (RPLGD). In: Gali-Muhtasib H, editor. Advances in cancer therapy. London: InTech; 2011. p. 553-68.

15. Pyone YY, Suriyapee $S$, Sanghangthum T, Oonsiri S, Tawonwong T. Determination of effective doses in image-guided radiation therapy system. J Phys Conf Ser 2016;694:012007. 